\title{
On Some Generalizations Bellman-Bihari Result for Integro-Functional Inequalities for Discontinuous Functions and Their Applications
}

\author{
Angela Gallo ${ }^{1}$ and Anna Maria Piccirillo ${ }^{2}$ \\ ${ }^{1}$ Department of Mathematics and Applications, "R.Caccioppoli" University of Naples "Federico II", \\ Claudio street 21, 80125 Naples, Italy \\ ${ }^{2}$ Department of Civil Engineering, Second University of Naples, Roma, street 21, 81100 Caserta, Italy \\ Correspondence should be addressed to Angela Gallo, angallo@unina.it
}

Received 22 December 2008; Revised 21 April 2009; Accepted 28 May 2009

Recommended by Juan J. Nieto

We present some new nonlinear integral inequalities Bellman-Bihari type with delay for discontinuous functions (integro-sum inequalities; impulse integral inequalities). Some applications of the results are included: conditions of boundedness (uniformly), stability by Lyapunov (uniformly), practical stability by Chetaev (uniformly) for the solutions of impulsive differential and integrodifferential systems of ordinary differential equations.

Copyright (C) 2009 A. Gallo and A. M. Piccirillo. This is an open access article distributed under the Creative Commons Attribution License, which permits unrestricted use, distribution, and reproduction in any medium, provided the original work is properly cited.

\section{Introduction}

The first generalizations of the Bihari result for discontinuous functions which satisfy nonlinear impulse inequality (integro-sum inequality) are connected with such types of inequalities:

(a)

$$
v(t) \leq c+\int_{t_{0}}^{t} p(\tau) v^{m}(\tau) d \tau+\sum_{t_{0}<t_{i}<t} \beta_{i} v\left(t_{i}-0\right), \quad m>0, m \neq 1
$$

(b)

$$
v(t) \leq c+\int_{t_{0}}^{t} p(\tau) \varphi(v(\tau)) d \tau+\sum_{t_{0}<t_{i}<t} \beta_{i} v\left(t_{i}-0\right),
$$


Which are studied in the publications by Bainov, Borysenko, Iovane, Laksmikantham, Leela, Martynyuk, Mitropolskiy, Samoilenko ([1-13]), and in many others. In these investigations the method of integral inequalities for continuous functions is generalized to the case of piecewise continuous (one-dimensional inequalities) and discontinuous (multidimensional inequalities) functions.

For the generalization of the integral inequalities method for discontinuous functions and for their applications to qualitative analysis of impulsive systems: existence, uniqueness, boundedness, comparison, stability, and so forth. We refer to the results $[2-5,12,14]$ and for periodic boundary value problems we cite [15-17]. More recently, a novel variational approach appeared in [18]. This approach to impulsive differential equations also used the critical point theory for the existence of solutions of a nonlinear Dirichlet impulsive problem and in [19] some new comparison principles and the monotone iterative technique to establish a more general existence theorem for a periodic boundary value problem. Reference [20] is very interesting in that it gives a complete overview of the state-of-the-art of the impulsive differential, inclusions.

In this paper, in Section 2, we investigate new analogies Bihari results for piece-wise continuous functions and, in Section 3, the conditions of boundedness, stability, pract-ical stability of the solutions of nonlinear impulsive differential and integro-differential systems.

\section{General Bihari Theorems for Integro-Functional Inequalities for Discontinuous Functions}

Let us consider the class $\wp$ of continuous functions $p: R \rightarrow R, p(t) \leq t, \lim _{|t| \rightarrow \infty} p(t)=\infty(p=$ $p(t)$ is the delaying argument). The following holds.

Theorem 2.1. (a) Let one suppose that for $x \geq x_{0}$ the following integro-sum functional inequality holds:

$$
u(x) \leq \varphi(x)+q(x) \int_{x_{i}}^{x} f(\tau) W(u(p(\tau))) d \tau+\sum_{x_{0}<x_{i}<x} \beta_{i} u^{m}\left(x_{i}-0\right)
$$

where $q(x) \geq 1, \varphi(x)$ is a positive nondecreasing function, $\boldsymbol{\beta}_{\mathbf{i}}=$ const $\geq 0, f: R_{+} \rightarrow R_{+}, m=$ const $>0$; function $u(x)$ is a nonnegative piecewise-continuous, with I-st kind of discontinuities in the points $x_{i}: x_{0}<x_{1}<\cdots \lim _{n \rightarrow \infty} x_{n}=\infty, p(t)$ belongs to the class $\wp$.

(b) Function $W(x)$ satisfies such conditions:

(i) $W(\gamma \beta) \leq W(\gamma) W(\beta)$;

(ii) $W: R_{+} \rightarrow R_{+}, W(0)=0$;

(iii) $W$ is nondecreasing.

Then for arbitrary $x \in] x_{0}, \infty[$ the next estimate holds:

$$
\begin{gathered}
\left.u(x) \leq \varphi(x) q(x) G_{i}^{-1}\left[\int_{x_{i}}^{x} \frac{f(\tau)}{\varphi(\tau)} W[\varphi(p(\tau)) q(p(\tau))] d \tau\right] \text { for } x \in\right] x_{i}, x_{i+1}[ \\
\int_{x_{i}}^{x} \frac{f(\tau)}{\varphi(\tau)} W[\varphi(p(\tau)) q(p(\tau))] d \tau \in \operatorname{Dom}\left(G_{i}^{-1}\right)
\end{gathered}
$$


Boundary Value Problems

$$
\begin{gathered}
G_{0}(u)=\int_{1}^{u} \frac{d \sigma}{W(\sigma)}, \\
G_{i}(u)=\int_{c_{i}}^{u} \frac{d \sigma}{W(\sigma)}, \quad i=1,2, \ldots, \\
c_{i}=\left(1+\beta_{i} \varphi^{m-1}\left(x_{i}\right) q^{m}\left(x_{i}-0\right)\right) G_{i-1}^{-1}\left(\int_{x_{i-1}}^{x_{i}} \frac{f(\tau)}{\varphi(\tau)} W[\varphi(p(\tau)) q(p(\tau))] d \tau\right), \\
i=1,2, \ldots \text { if } m \in] 0,1], \forall x \geq x_{0}, \\
c_{i}=\left(1+\beta_{i} \varphi^{m-1}\left(x_{i}\right) q^{m}\left(x_{i}-0\right)\right)\left[G_{i-1}^{-1}\left(\int_{x_{i-1}}^{x_{i}} \frac{f(\tau)}{\varphi(\tau)} W[\varphi(p(\tau)) q(p(\tau))] d \tau\right)\right]^{m}, \\
i=1,2, \ldots \text { if } m \geq 1, \forall x \geq x_{0} .
\end{gathered}
$$

Proof. It follows from inequality (2.1)

$$
\begin{aligned}
\frac{u(x)}{\varphi(x)} & \leq 1+q(x) \int_{x_{0}}^{x} \frac{f(\tau) W(u(p(\tau)))}{\varphi(\tau)} d \tau+\sum_{x_{0}<x_{i}<x} \beta_{i} \frac{u^{m}\left(x_{i}-0\right)}{\varphi(x)} \\
& \leq q(x)\left\{1+\int_{x_{0}}^{x} \frac{f(\tau)}{\varphi(\tau)} W(u(p(\tau))) d \tau+\sum_{x_{0}<x_{i}<x} \beta_{i} \varphi^{m-1}\left(x_{i}-0\right)\left[\frac{u\left(x_{i}-0\right)}{\varphi\left(x_{i}-0\right)}\right]^{m}\right\} .
\end{aligned}
$$

Denoting by

$$
\begin{aligned}
& u^{*}(x)=1+\int_{x_{0}}^{x} \frac{f(\tau)}{\varphi(\tau)} W(u(p(\tau))) d \tau+\sum_{x_{0}<x_{i}<x} \beta_{i} \varphi^{m-1}\left(x_{i}-0\right)\left[\frac{u\left(x_{i}-0\right)}{\varphi\left(x_{i}-0\right)}\right]^{m}, \\
& u^{*}(x)=1 \text { for } x=x_{0},
\end{aligned}
$$

then

$$
\begin{aligned}
& u\left(x_{i}-0\right) \leq \varphi\left(x_{i}-0\right) q\left(x_{i}-0\right) u^{*}\left(x_{i}-0\right) \\
& u(x) \leq \varphi(x) q(x) u^{*}(x) \\
& u(p(\tau)) \leq \varphi(p(\tau)) q(p(\tau)) u^{*}(p(\tau)) \leq \varphi(p(\tau)) q(p(\tau)) u^{*}(\tau), \\
& \Longrightarrow u^{*}(x) \leq 1+\int_{x_{0}}^{x} \frac{f(\tau)}{\varphi(\tau)} W[\varphi(p(\tau)) q(p(\tau))] W\left(u^{*}(\tau)\right) d \tau \\
& \quad+\sum_{x_{0}<x_{i}<x} \beta_{i} \varphi^{m-1}\left(x_{i}-0\right) q^{m}\left(x_{i}-0\right) u^{* m}\left(x_{i}-0\right) .
\end{aligned}
$$


Let us consider the interval $I_{1}=\left[x_{0}, x_{1}[\right.$. Then

$$
\begin{aligned}
u^{*}(x) \leq & G_{0}^{-1}\left(\int_{x_{0}}^{x} \frac{f(\tau)}{\varphi(\tau)} W[\varphi(p(\tau)) q(p(\tau))] d \tau\right), \\
& \text { if only } \int_{x_{0}}^{x} \frac{f(\tau)}{\varphi(\tau)} W[\varphi(p(\tau)) q(p(\tau))] d \tau \in \operatorname{Dom}\left(G_{0}^{-1}\right),
\end{aligned}
$$

where $G_{0}(\xi)=\int_{1}^{\xi}(d \tau / W(\tau))$. So it results in

$$
u(x) \leq \varphi(x) q(x) G_{0}^{-1}\left[\int_{x_{0}}^{x} \frac{f(\tau)}{\varphi(\tau)} W[\varphi(p(\tau)) q(p(\tau))] d \tau\right]
$$

and estimate (2.2) is valid in $I_{1}$.

Let us suppose that for $x \in I_{k}=\left\lfloor x_{k-1}, x_{k}\right\rfloor, k=2,3, \ldots$ estimate (2.2) is fulfilled. Then for every $x \in I_{k+1}$ we have

$$
\begin{aligned}
& u^{*}(x) \leq G_{k}^{-1}\left(\int_{x_{k}}^{x} \frac{f(\tau)}{\varphi(\tau)} W[\varphi(p(\tau)) q(p(\tau))] d \tau\right) \\
& \quad \text { with } \int_{x_{k}}^{x} \frac{f(\tau)}{\varphi(\tau)} W[\varphi(p(\tau)) q(p(\tau))] d \tau \in \operatorname{Dom}\left(G_{k}^{-1}\right),
\end{aligned}
$$

where $G_{k}(\xi)$ is determined from (2.3)-(2.5).

Taking into account such inequality

$$
u(x) \leq \varphi(x) q(x) u^{*}(x)
$$

we obtain estimate (2.2) for every $x \in\left[x_{0}, \infty[\right.$.

Let us consider the class $\mathfrak{I}$ of functions $f$ such that

(i) $f(x)$-positive, continuous, nondecreasing for $x>0$;

(ii) $\forall u \geq 1, v>0 \Rightarrow u^{-1} f(v)<f\left(u^{-1} v\right)$;

(iii) $f(0)=0$.

The following result is proved.

Theorem 2.2. Suppose that the part (a) of Theorem 2.1 is valid and function $W:[0, \infty[\rightarrow[0, \infty[$ belongs to the class $\mathfrak{I}$. Then for arbitrary $x_{0} \leq x \leq x^{*}$ such estimate holds:

$$
u(x) \leq \varphi(x) q(x) G_{i}^{*-1}\left[\int_{x_{i}}^{x} f(\tau) q(p(\tau)) d \tau\right] \text { for } I_{i}=\left[x_{i}, x_{i+1}[, i=0,1, \ldots\right.
$$


where

$$
\begin{aligned}
G_{0}^{*}(\eta) & =\int_{1}^{\eta} \frac{d \sigma}{W(\sigma)}, \quad G_{i}^{*}(\eta)=\int_{c_{i}^{*}}^{\eta} \frac{d \sigma}{W(\sigma)} i=1,2, \ldots, \\
c_{i}^{*} & \left.\left.=\left(1+\beta_{i} \varphi^{m-1}\left(x_{i}\right) q^{m}\left(x_{i}\right)\right) G_{i-1}^{*}-1\left(\int_{x_{i-1}}^{x_{i}} f(\tau) q(p(\tau)) d \tau\right) \quad \text { if } m \in\right] 0,1\right], \\
c_{i}^{*} & =\left(1+\beta_{i} \varphi^{m-1}\left(x_{i}\right) q^{m}\left(x_{i}\right)\right)\left[G_{i-1}^{*}-1\left(\int_{x_{i-1}}^{x_{i}} f(\tau) q(p(\tau)) d \tau\right)\right]^{m} \quad \text { if } m \geq 1,
\end{aligned}
$$

and $x^{*}=\sup _{x}\left\{\int_{x_{i-1}}^{x} f(\tau) q(p(\tau)) d \tau \in \operatorname{Dom}\left(G_{i-1}^{*}{ }^{-1}\right)\right\}, i=1,2, \ldots$

Proof. By using the previous theorem we have $u(x) \leq \varphi(x) g(x) u^{*}(x), u^{*}(x)=1 x=x_{0}$. On the interval $I_{1}$

$$
\frac{d u^{*}(x)}{d x}=\frac{f(x)}{\varphi(x)} W(u(p(x)))
$$

Then

$$
\begin{aligned}
u(p(x)) & \leq \varphi(p(x)) q(p(x)) u^{*}(p(x)) \leq \varphi(x) q(p(x)) u^{*}(x) \\
\frac{d u^{*}(x)}{d x} & \leq \frac{f(x)}{\varphi(x)} W\left(q(p(x)) \varphi(x) u^{*}(x)\right) \\
& \leq \frac{f(x) q(p(x))}{\varphi(x) q(p(x))} W\left(q(p(x)) \varphi(x) u^{*}(x)\right) \\
& \leq f(x) q(p(x)) W\left(u^{*}(x)\right)
\end{aligned}
$$

Taking into account estimate (2.16), we obtain

$$
\begin{gathered}
\int_{x_{0}}^{x} \frac{u^{* \prime}(\sigma)}{W\left(u^{*}(\sigma)\right)} d \sigma \leq \int_{x_{0}}^{x} f(\tau) q(p(\tau)) d \tau, \\
\int_{x_{0}}^{x} \frac{u^{* \prime}(\sigma)}{W\left(u^{*}(\sigma)\right)} d \sigma=\int_{u^{*}\left(x_{0}\right)}^{u^{*}(x)} \frac{d u}{W(u)}=G_{0}^{*}\left(u^{*}(x)\right)-G_{0}^{*}\left(u^{*}\left(x_{0}\right)\right), \\
u^{*}\left(x_{0}\right)=1, \quad u^{*}(x) \geq 1, \quad G_{0}^{*}\left(u^{*}\left(x_{0}\right)\right)=G_{0}^{*}(1)=0, \\
G_{0}^{*}\left(u^{*}(x)\right) \leq \int_{x_{0}}^{x} f(\tau) q(p(\tau)) d \tau .
\end{gathered}
$$


Then in $I_{1}$ we have

$$
u(x) \leq \varphi(x) q(x) G_{0}^{*-1}\left[\int_{x_{0}}^{x} f(\tau) q(p(\tau)) d \tau\right] \text { if only } \int_{x_{0}}^{x} f(\tau) q(p(\tau)) d \tau \in \operatorname{Dom}\left(G_{0}^{*-1}\right)
$$

As in the previously theorem, the proof is completed by using the inductive method.

The following result is easily to obtain

Theorem 2.3. Suppose that for $x \geq x_{0}$ the next inequality holds:

$$
\begin{aligned}
u(x) \leq & u_{0}+q(x)\left[\int_{x_{0}}^{x} f(s) u(p(s)) d s+\int_{x_{0}}^{x} f(s)\left(\int_{x_{0}}^{x} g(\tau) u(p(\tau)) d \tau\right) d s\right] \\
& +\int_{x_{0}}^{x} h(s) W(u(\sigma(s))) d s+\sum_{x_{0}<x_{i}<x} \beta_{i} u^{m}\left(x_{i}-0\right),
\end{aligned}
$$

where functions $u(x), f(x), q(x), g(x), h(x), p(x), \sigma(x)$ are real nonnegative for $x \geq x_{0}>$ $0, p(x), \sigma(x) \in \mathfrak{I}, q(x) \geq 1, \beta_{i} \geq 0$, function $W$ satisfies conditions (i),...,(iii) of Theorem 2.1.

Then for $x \geq x_{0}$ it results in

$$
\begin{aligned}
& u(x) \leq \prod_{x_{0}<x_{i}<x}\left(1+\beta_{i} q^{m}\left(x_{i}\right) u_{0}^{m-1}\right) \exp \left(\int_{x_{0}}^{x} q(p(\tau))[f(\tau)+g(\tau)] d \tau\right) \\
& \cdot \psi_{0}^{-1}\left(\int_{x_{0}}^{x} h(\tau) W\left[\prod_{x_{0}<x_{i}<\sigma(\tau)}\left(1+\beta_{i} q^{m}\left(x_{i}\right) u_{0}^{m-1}\right)\right] W\right. \\
&\left.\left.\left.\times\left[q(\sigma(\tau)) \exp \left(\int_{x_{0}}^{\sigma(\tau)} q(p(s))[f(s)+g(s)] d s\right)\right] d \tau\right), \quad \text { if } m \in\right] 0,1\right] \\
& \int_{x_{0}}^{x} h(\tau) W\left[\prod_{x_{0}<x_{i}<\sigma(\tau)}\left(1+\beta_{i} q^{m}\left(x_{i}\right) u_{0}^{m-1}\right)\right] W \\
& \times\left[q(\sigma(\tau)) \exp \left(\int_{x_{0}}^{\sigma(\tau)} q(p(s))[f(s)+g(s)] d s\right)\right] d \tau \in \operatorname{Dom}\left(\psi_{0}^{-1}\right),
\end{aligned}
$$


where $\psi_{0}(u)=\int_{u_{0}}^{u}(d v / W(v))$;

$$
\begin{aligned}
& u(x) \leq \prod_{x_{0}<x_{i}<x}\left(1+\beta_{i} q^{m}\left(x_{i}\right) u_{0}^{m-1}\right) \exp \left(m \int_{x_{0}}^{x} q(p(\tau))[f(\tau)+g(\tau)]\right) \\
& \cdot \psi_{0}^{-1}\left(\int_{x_{0}}^{x} h(\tau)\left[\prod_{x_{0}<x_{i}<\sigma(\tau)}\left(1+\beta_{i} q^{m}\left(x_{i}\right) u_{0}^{m-1}\right)\right]\right. \\
&\left.\cdot W\left[q(\sigma(\tau)) \exp \left(m \int_{x_{0}}^{\sigma(\tau)} q(p(s))[f(s)+g(s)] d s\right)\right] d \tau\right), \quad \text { if } m \geq 1, \\
& \int_{x_{0}}^{x} h(\tau) W\left[\prod_{x_{0}<x_{i}<\sigma(\tau)}\left(1+\beta_{i} q^{m}\left(x_{i}\right) u_{0}^{m-1}\right)\right] W \\
& \times\left[q(\sigma(\tau)) \exp \left(m \int_{x_{0}}^{\sigma(\tau)} q(p(s))[f(s)+g(s)] d s\right)\right] d \tau \in \operatorname{Dom}\left(\psi_{0}^{-1}\right) .
\end{aligned}
$$

The proof the same procedure as that of (Iovane [21, Theorems 2.1 and 3.1]).

\section{Corollary 2.4. Suppose that}

(a) $m=1$, then the result of Theorem 2.1 coincides with the result [22, Theorem 3.7.1, page 232];

(b) $m=1, \varphi(x)=c, q(x)=1, p(t)=t$, then the result of Theorem 2.1 coincides with result [12, Proposition 2.3, page 2143];

(c) $q(x)=1, W(u)=u, p(t)=t$, then one obtains the analogy of Gronwall-Bellman result for discontinuous functions [23, Lemma 1] and estimate (2.2) reduces in the following form:

$$
\begin{aligned}
& \left.\left.u(x) \leq \varphi(x) \prod_{x_{0}<x_{i}<x}\left(1+\beta_{i} \varphi^{m-1}\left(x_{i}\right)\right) \exp \left(\int_{x_{0}}^{x} f(\tau) d \tau\right) \quad \text { if } m \in\right] 0,1\right], \forall x \geq x_{0}, \\
& u(x) \leq \varphi(x) \prod_{x_{0}<x_{i}<x}\left(1+\beta_{i} \varphi^{m-1}\left(x_{i}\right)\right) \exp \left(m \int_{x_{0}}^{x} f(\tau) d \tau\right) \text { if } m \geq 1, \forall x \geq x_{0} .
\end{aligned}
$$

(d) $q(x)=1, W(u)=u$, then one obtains the result [21, Theorem 2.1] and estimate (2.2) are as follows:

$$
\begin{aligned}
& \left.\left.u(x) \leq \varphi(x) \prod_{x_{0}<x_{i}<x}\left(1+\beta_{i} \varphi^{m-1}\left(x_{i}\right)\right) \exp \left(\int_{x_{0}}^{x} f(\tau) \frac{\varphi(p(\tau))}{\varphi(\tau)} d \tau\right), \quad \text { if } m \in\right] 0,1\right], \forall x \geq x_{0} ; \\
& u(x) \leq \varphi(x) \prod_{x_{0}<x_{i}<x}\left(1+\beta_{i} \varphi^{m-1}\left(x_{i}\right)\right) \exp \left(m \int_{x_{0}}^{x} f(\tau) \frac{\varphi(p(\tau))}{\varphi(\tau)} d \tau\right) \quad \text { if } m \geq 1, \forall x \geq x_{0} .
\end{aligned}
$$


(e) $q(x)=1, W(u)=u^{m}, m>0, p(t)=t$, then one obtains the analogy of Bihari result for discontinuous functions [23, Lemma 2] and estimate (2.2) reduces as follows are reduced:

$$
\begin{gathered}
u(x) \leq \varphi(x) \prod_{x_{0}<x_{i}<x}\left(1+\beta_{i} \varphi^{m-1}\left(x_{i}\right)\right)\left[1+(1-m) \int_{x_{0}}^{x} \varphi^{m-1}(\tau) f(\tau) d \tau\right]^{1 /(1-m)}, \\
\text { if } 0<m<1, \forall x \geq x_{0}, \\
u(x) \leq \varphi(x) \prod_{x_{0}<x_{i}<x}\left(1+\beta_{i} m \varphi^{m-1}\left(x_{i}\right)\right)\left[1-(m-1)\left[\prod_{x_{0}<x_{i}<x}\left(1+\beta_{i} m \varphi^{m-1}\left(x_{i}\right)\right)\right]^{m-1}\right. \\
\left.\times \int_{x_{0}}^{x} \varphi^{m-1}(\tau) f(\tau) d \tau\right]^{-1 /(m-1)} \quad \forall x \geq x_{0},
\end{gathered}
$$

such that

$$
\int_{x_{0}}^{x} \varphi^{m-1}(\tau) f(\tau) d \tau \leq \frac{1}{m}, \quad m>1, \quad \prod_{x_{0}<x_{i}<x}\left(1+\beta_{i} \varphi^{m-1}\left(x_{i}\right)\right)<\left(1+\frac{1}{m-1}\right)^{1 /(m-1)} .
$$

(f) $W(u)=u^{m}, m>0$, then estimate (2.2) reduces as follows (see [21, Theorem 2.2]):

$$
\begin{aligned}
u(x) \leq & \varphi(x) q(x) \prod_{x_{0}<x_{i}<x}\left(1+\beta_{i} \varphi^{m-1}\left(x_{i}\right) q^{m}\left(x_{i}\right)\right) \\
\times & {\left[1+(1-m) \int_{x_{0}}^{x} \varphi^{m-1}(\tau) f(\tau) q^{m}(p(\tau))\left[\frac{\varphi(p(\tau))}{\varphi(\tau)}\right]^{m} d \tau\right]^{1 /(1-m)} } \\
\text { if } 0<m<1, \forall x \geq x_{0}, & \\
u(x) \leq & \varphi(x) q(x) \prod_{x_{0}<x_{i}<x}\left(1+\beta_{i} m \varphi^{m-1}\left(x_{i}\right) q^{m}\left(x_{i}\right)\right) \\
& \times\left\{1-(m-1)\left[\prod_{x_{0}<x_{i}<x}\left(1+\beta_{i} m \varphi^{m-1}\left(x_{i}\right) q^{m}\left(x_{i}\right)\right)\right]^{m-1}\right. \\
& \left.\times \int_{x_{0}}^{x} \varphi^{m-1}(\tau) f(\tau) q^{m}(p(\tau))\left[\frac{\varphi(p(\tau))}{\varphi(\tau)}\right]^{m} d \tau\right\}^{-1 /(m-1)} \forall x \geq x_{0}
\end{aligned}
$$


Boundary Value Problems

such that

$$
\begin{gathered}
\int_{x_{0}}^{x} \varphi^{m-1}(\tau) f(\tau) q^{m}(p(\tau))\left[\frac{\varphi(p(\tau))}{\varphi(\tau)}\right]^{m} d \tau \leq \frac{1}{m}, \quad m>1, \\
\prod_{x_{0}<x_{i}<x}\left(1+\beta_{i} m \varphi^{m-1}\left(x_{i}\right) q^{m}\left(x_{i}\right)\right)<\left(1+\frac{1}{m-1}\right)^{-1 /(m-1)} .
\end{gathered}
$$

(g) Suppose that in Theorem $2.3 q(x)=1, W(u)=u, \sigma(s)=p(s)=s$, then estimates (2.20), (2.21) reduce as shown:

$$
\begin{aligned}
& \left.\left.u(x) \leq u_{0} \prod_{x_{0}<x_{i}<x}\left(1+\beta_{i} u_{0}^{m-1}\left(x_{i}\right)\right) \exp \left[\int_{x_{0}}^{x}[f(\xi)+g(\xi)+h(\xi)] d \xi\right] \quad \text { if } m \in\right] 0,1\right], \forall x \geq x_{0} \\
& u(x) \leq u_{0} \prod_{x_{0}<x_{i}<x}\left(1+\beta_{i} u_{0}^{m-1}\left(x_{i}\right)\right) \exp \left[m \int_{x_{0}}^{x}[f(\xi)+g(\xi)+h(\xi)] d \xi\right] \quad \text { if } m \geq 1, \forall x \geq x_{0}
\end{aligned}
$$

which coincide with result of [21, Theorem 3.1] for $h(t)=u_{0}$.

\section{Applications}

Let us consider the following system of differential equations

$$
\begin{gathered}
\frac{d x}{d t}=F(t, x), \quad t \neq t_{i} \\
\left.\Delta x\right|_{t=t_{i}}=I_{i}(x)
\end{gathered}
$$

where $x \in \mathfrak{R}^{n}, F \in \mathfrak{R}^{n}, I_{i}(x) \in \mathfrak{R}^{n} \quad(i=1,2, \ldots), t \geq t_{0} \geq 0, \lim _{i \rightarrow \infty} t_{i}=\infty, t_{i-1}<t_{i}$ for all $i=$ $1,2, \ldots$

Let us assume that $F(t, x)$ and $I_{i}(x)$ are defined in the domain $D=\{(t, x): t \in \mathfrak{I}=$ $\left.\left[t_{0}, T\right], T \leq \infty,\|x\| \leq h\right\}$ and satisfy such conditions:

(a) $\|F(t, x)\| \leq f(t) W(\|x\|), f: \Re_{+} \rightarrow \Re_{+}$,

W satisfies conditions (i)-(iii) of Theorem 2.1;

(b) $\left\|I_{i}(x)\right\| \leq \beta_{i}\|x\|^{m}, \beta_{i}=$ const $>0, m>0$.

Consider $x(t)=x\left(t, t_{0}, x_{0}\right)$ the solution of Cauchy problem for system (3.1). Then

$$
x\left(t, t_{0}, x_{0}\right)=x_{0}+\int_{t_{0}}^{t} F\left(\tau, x\left(\tau, t_{0}, x_{0}\right)\right) d \tau+\sum_{t_{0}<t_{i}<t} I_{i}\left(x\left(t_{i}-0, t_{0}, x_{0}\right)\right),
$$


from which it follows

$$
\left\|x\left(t, t_{0}, x_{0}\right)\right\| \leq\left\|x_{0}\right\|+\int_{t_{0}}^{t} f(\tau) W\left(\left\|x\left(\tau, t_{0}, x_{0}\right)\right\|\right) d \tau+\sum_{t_{0}<t_{i}<t} \beta_{i}\left\|x\left(t_{i}-0, t_{0}, x_{0}\right)\right\|^{m} .
$$

By using the result of Theorem 2.1 and estimate (2.2) we obtain

$$
\begin{aligned}
& \left.\left\|x\left(t, t_{0}, x_{0}\right)\right\| \leq\left\|x_{0}\right\| G_{i}^{-1}\left[\int_{x_{i}}^{x} f(\tau) \frac{W\left(\left\|x_{0}\right\|\right)}{\left\|x_{0}\right\|} d \tau\right] \text { for } x \in\right] x_{i}, x_{i+1}[ \\
& \int_{x_{i}}^{x} f(\tau) \frac{W\left(\left\|x_{0}\right\|\right)}{\left\|x_{0}\right\|} d \tau \in \operatorname{Dom}\left(G_{i}^{-1}\right),
\end{aligned}
$$

where

$$
\begin{gathered}
G_{0}(u)=\int_{1}^{u} \frac{d \sigma}{W(\sigma)}, \quad G_{i}(u)=\int_{c_{i}}^{u} \frac{d \sigma}{W(\sigma)}, \quad i=1,2, \ldots, \\
c_{i}=\left(1+\beta_{i}\left\|x_{0}\right\|^{m-1}\right) G_{i-1}^{-1}\left(\int_{x_{i-1}}^{x_{i}} f(\tau) \frac{W\left(\left\|x_{0}\right\|\right)}{\left\|x_{0}\right\|} d \tau\right), \\
i=1,2, \ldots \quad \text { if } m \in] 0,1], \forall x \geq x_{0}, \\
c_{i}=\left(1+\beta_{i}\left\|x_{0}\right\|^{m-1}\right)\left[G_{i-1}^{-1}\left(\int_{x_{i-1}}^{x_{i}} f(\tau) \frac{W\left(\left\|x_{0}\right\|\right)}{\left\|x_{0}\right\|} d \tau\right)\right]^{m}, \\
i=1,2, \ldots \quad \text { if } m \geq 1, \forall x \geq x_{0} .
\end{gathered}
$$

Let us consider some particular cases of $W$.

If $W(u)=u, m=1$, estimate (3.4) is reduced in such form

$$
\left\|x\left(t, t_{0}, x_{0}\right)\right\| \leq\left\|x_{0}\right\| \prod_{t_{0}<t_{i}<t}\left(1+\beta_{i}\right) \exp \left[\int_{t_{0}}^{t} f(\tau) d \tau\right] .
$$

Then such result holds.

Proposition 3.1. Let the following conditions be fulfilled for system (3.1) :

(i) $\|F(t, x)\| \leq f(t)\|x\| ;$

(ii) $\left\|I_{i}(x)\right\| \leq \beta_{i}\|x\|$;

(iii) $\exists m_{1}\left(t_{0}\right)=$ const. $>0: \prod_{t_{0}<t_{i}<t}\left(1+\beta_{i}\right) \leq m_{1}\left(t_{0}\right)<\infty$;

(iv) $\exists m_{2}\left(t_{0}\right)=$ const. $>0: \int_{t_{0}}^{t} f(\tau) d \tau \leq m_{2}\left(t_{0}\right)<\infty, \forall t \geq t_{0}$. 
Then one has:

(a) All solutions of system (3.1) are bounded (uniformly, if $m_{i}\left(t_{0}\right)$ are independent of $t_{0}$ ) and such estimate is valid:

$$
\left\|x\left(t, t_{0}, x_{0}\right)\right\| \leq m_{1}\left(t_{0}\right) \exp \left[m_{2}\left(t_{0}\right)\right]\left\|x_{0}\right\|
$$

(b) The trivial solution of system (3.1) is stable by Lyapunov (uniformly stable relative $t_{0}$, if $\left.m_{i}\left(t_{0}\right)=m_{i}, i=1,2\right)$.

Remark 3.2. If conditions I-IV of Proposition 3.1 are valid and $\lambda / \Lambda<\left(m_{1}\left(t_{0}\right) \exp \left[m_{2}\left(t_{0}\right)\right]\right)^{-1}$, then the trivial solution is $(\lambda, \Lambda, \mathfrak{I})$-stable by Chetaev (uniformly $(\lambda, \Lambda, \mathfrak{I})$-stable, if $m_{i}\left(t_{0}\right)$, $i=1,2$ is independent of $\left.t_{0}\right)$.

If $W(u)=u^{l}, l \neq 1, m=1$ the estimate (3.4) is reduced in such form

$$
\begin{aligned}
\left\|x\left(t, t_{0}, x_{0}\right)\right\| \leq & \prod_{t_{0}<t_{i}<t}\left(1+\beta_{i}\right)\left[\left\|x_{0}\right\|^{1-l}+(1-l) \int_{t_{0}}^{t} f(\tau) d \tau\right]^{1 /(1-l)} \quad \forall t \geq t_{0}, \text { if } 0<l<1, \\
\left\|x\left(t, t_{0}, x_{0}\right)\right\| \leq & \left\|x_{0}\right\| \prod_{t_{0}<t_{i}<t}\left(1+\beta_{i}\right) \\
& \times\left[1-(l-1)\left\|x_{0}\right\|^{l-1} \cdot\left[\prod_{t_{0}<t_{i}<t}\left(1+\beta_{i}\right)\right]^{-1} \int_{t_{0}}^{t} f(\tau) d \tau\right]^{-1 /(l-1)} \quad \forall t \geq t_{0}, \\
& \int_{t_{0}}^{t} f(\tau) d \tau<\left((l-1)\left[\left\|x_{0}\right\| \prod_{t_{0}<t_{i}<t}\left(1+\beta_{i}\right)\right]^{l-1}\right)^{-1}, \quad \text { if } l>1 .
\end{aligned}
$$

From estimate (3.8) the next propositions follow.

Proposition 3.3. Suppose that such conditions occur:

(a) $\|F(t, x)-F(t, y)\| \leq f(t)\|x-y\|^{l}, 0<l<1$ for all $x, y \in D$

(b) estimates ii-iv of Proposition 3.1 be fulfilled.

Then all the solutions of system (3.1) are bounded (uniformly if $m_{i}\left(t_{0}\right)=m_{i}, i=1,2$ ).

Remark 3.4. Suppose that conditions (a), (b) of Proposition 3.3 are valid and

$$
\lambda^{1-l}+(1-l) m_{2}\left(t_{0}\right)<\left[\frac{\Lambda}{m_{1}\left(t_{0}\right)}\right]^{1-l}
$$


Then trivial solution of system (3.1) is $(\lambda, \Lambda, \mathfrak{I})$-stable by Chetaev (uniformly if $m_{i}\left(t_{0}\right)$ is independent of $t_{0}$ ).

Proposition 3.5. Let conditions ii-iv of Proposition 3.1 be fulfilled for system (3.1), inequality (3.10) holds and

$$
\|F(t, x)\| \leq f(t)\|x\|^{l}, \quad l>1
$$

Then trivial solution of system (3.1) is stable by Lyapunov (uniformly if $m_{i}\left(t_{0}\right)=m_{i}, i=1,2$ ).

Remark 3.6. If $W(u)=u^{1}, 1>0$, and $m \neq 1$ the conditions of boundedness, stability, $(\lambda, \Lambda, \mathfrak{I})$ stability is investigated in [14, see Theorems 3.4-3.6]; the estimates of the solutions of system (3.1) with non-Lipschitz type of discontinuities are investigated in [23, see Proposition 1, Proposition 2].

Let us consider the following impulsive system of integro-differential equations:

$$
\begin{gathered}
\frac{d x}{d t}=F(t, x, K[x(t)]), \quad t \neq t_{i}, \\
\left.\Delta x\right|_{t=t_{i}}=I_{i}(x),
\end{gathered}
$$

where $x \in \mathfrak{R}^{n}, F \in \mathfrak{R}^{n}, I_{i}(x) \in \mathfrak{R}^{n}(i=1,2, \ldots)$ and defined in the domain $D, K[x(t)]=$ $\int_{t_{0}}^{t} k(t, \tau, x(\tau)) d \tau$.

We suppose that such conditions are valid:

(i) $\|F(t, x, y)\| \leq f(t)[\|x\|+\|y\|]$ for all $x, y \in D, f: \mathfrak{R}_{+} \rightarrow \mathfrak{R}_{+}$;

(ii) $\|k(t, s, x)\| \leq g(t)\|x\|$ for all $s \in\left[t_{0}, t\right], g: \Re_{+} \rightarrow \Re_{+}$;

(iii) $\left\|I_{i}(x)\right\| \leq \beta_{i}\|x\|^{m}$ for all $x, y \in D, \beta_{i}=$ const $>0, m>0 m \neq 1$.

It is easy to see that

$$
\begin{aligned}
&\left\|x\left(t, t_{0}, x_{0}\right)\right\| \leq\left\|x_{0}\right\|+\int_{t_{0}}^{t} f(\tau)\left\|x\left(\tau, t_{0}, x_{0}\right)\right\| d \tau \\
&+\int_{t_{0}}^{t} f(\tau)\left(\int_{t_{0}}^{\tau} g(\xi)\left\|x\left(\xi, t_{0}, x_{0}\right)\right\| d \xi\right) d \tau+\sum_{t_{0}<t_{i}<t} \beta_{i}\left\|x\left(t_{i}-0, t_{0}, x_{0}\right)\right\|^{m} \\
& \Longrightarrow\left\|x\left(t, t_{0}, x_{0}\right)\right\| \leq\left\|x_{0}\right\| \prod_{t_{0}<t_{i}<t}\left(1+\beta_{i}\left\|x_{0}\right\|^{m-1}\right) \exp \int_{t_{0}}^{t}[f(\xi)+g(\xi)] d \xi, \\
& \text { if } 0<m \leq 1, t \geq t_{0} \\
&\|x(t)\| \leq\left\|x_{0}\right\| \prod_{t_{0}<t_{i}<t}\left(1+\beta_{i}\left\|x_{0}\right\|^{m-1}\right) \exp \left(m \int_{t_{0}}^{t}[f(\xi)+g(\xi)] d \xi\right),
\end{aligned}
$$$$
\text { if } 0 m \geq 1, t \geq t_{0} \text {. }
$$

From estimate (3.15) such result follows. 
Proposition 3.7. Let one suppose that for system (3.13) conditions (i)-(iii) take place for $m>1$ and the following estimates are fulfilled:

(a) $\exists m_{3}\left(t_{0}\right)=$ const. $>0: \prod_{t_{0}<t_{i}<t}\left(1+\beta_{i}\left\|x_{0}\right\|^{m-1}\right) \leq m_{3}\left(t_{0}\right)<\infty$;

(b) $\exists m_{4}\left(t_{0}\right)=$ const. $>0: \int_{t_{0}}^{t}[f(\xi)+g(\xi)] d \xi \leq m_{4}\left(t_{0}\right)<\infty$ for all $t \geq t_{0}$.

Then we have:

(i) All solutions of system (3.13) are bounded and satisfy the estimate:

$$
\|x(t)\| \leq m_{3}\left(t_{0}\right) \exp \left[m_{4}\left(t_{0}\right)\right]\left\|x_{0}\right\| .
$$

(ii) The trivial solution of system (3.13) is stable by Lyapunov (uniformly, if $m_{i}\left(t_{0}\right)=m_{i}$, $i=$ $3,4)$.

(iii) The trivial solution of system (3.13) is $(\lambda, \Lambda, \mathfrak{I})$-stable by Chetaev (uniformly if $m_{i}\left(t_{0}\right)$ is independent of $\left.t_{0}\right)$ and $m_{3}\left(t_{0}\right) \exp \left[m_{4}\left(t_{0}\right)\right]<\Lambda / \lambda$.

\section{References}

[1] D. Banov and P. Simeonov, Integral Inequalities and Applications, vol. 57 of Mathematics and Its Applications, Kluwer Academic Publishers, Dordrecht, The Netherlands, 1992.

[2] S. D. Borysenko, G. Iovane, and P. Giordano, "Investigations of the properties motion for essential nonlinear systems perturbed by impulses on some hypersurfaces," Nonlinear Analysis: Theory, Methods E Applications, vol. 62, no. 2, pp. 345-363, 2005.

[3] S. D. Borysenko, M. Ciarletta, and G. Iovane, "Integro-sum inequalities and motion stability of systems with impulse perturbations," Nonlinear Analysis: Theory, Methods E Applications, vol. 62, no. 3, pp. 417-428, 2005.

[4] S. Borysenko and G. Iovane, "About some new integral inequalities of Wendroff type for discontinuous functions," Nonlinear Analysis: Theory, Methods E Applications, vol. 66, no. 10, pp. 21902203, 2007.

[5] S. C. Hu, V. Lakshmikantham, and S. Leela, "Impulsive differential systems and the pulse phenomena," Journal of Mathematical Analysis and Applications, vol. 137, no. 2, pp. 605-612, 1989.

[6] V. Lakshmikantham and S. Leela, Differential and Integral Inequalities, Theory and Applications, Academic Press, New York, NY, USA, 1969.

[7] V. Lakshmikantham, S. Leela, and M. Mohan Rao Rama, "Integral and integro-differential inequalities," Applicable Analysis, vol. 24, no. 3, pp. 157-164, 1987.

[8] V. Lakshmikantham, D. D. Bainov, and P. S. Simeonov, Theory of Impulsive Differential Equations, vol. 6 of Series in Modern Applied Mathematics, World Scientific, Teaneck, NJ, USA, 1989.

[9] A. A. Martyhyuk, V. Lakshmikantham, and S. Leela, Stability of Motion: the Method of Integral Inequalities , Naukova Dumka, Kyiv, Russia, 1989.

[10] Yu. A. Mitropolskiy, S. Leela, and A. A. Martynyuk, "Some trends in V. Lakshmikantham's investigations in the theory of differential equations and their applications," Differentsial'nye Uravneniya, vol. 22, no. 4, pp. 555-572, 1986.

[11] Yu. A. Mitropolskiy, A. M. Samoilenko, and N. Perestyuk, "On the problem of substantiation of overoging method for the second equations with impulse effect," Ukrainskii Matematicheskii Zhurnal, vol. 29, no. 6, pp. 750-762, 1977.

[12] Yu. A. Mitropolskiy, G. Iovane, and S. D. Borysenko, "About a generalization of Bellman-Bihari type inequalities for discontinuous functions and their applications," Nonlinear Analysis: Theory, Methods E Applications, vol. 66, no. 10, pp. 2140-2165, 2007.

[13] A. M. Samoilenko and N. Perestyuk, Differential Equations with Impulse Effect, Visha Shkola, Kyiv, Russia, 1987. 
[14] A. Gallo and A. M. Piccirillo, "About new analogies of Gronwall-Bellman-Bihari type inequalities for discontinuous functions and estimated solutions for impulsive differential systems," Nonlinear Analysis: Theory, Methods E Applications, vol. 67, no. 5, pp. 1550-1559, 2007.

[15] J. J. Nieto, "Impulsive resonance periodic problems of first order," Applied Mathematics Letters, vol. 15, no. 4, pp. 489-493, 2002.

[16] J. J. Nieto, "Basic theory for nonresonance impulsive periodic problems of first order," Journal of Mathematical Analysis and Applications, vol. 205, no. 2, pp. 423-433, 1997.

[17] J. J. Nieto, "Periodic boundary value problems for first-order impulsive ordinary differential equations," Nonlinear Analysis: Theory, Methods E Applications, vol. 51, no. 7, pp. 1223-1232, 2002.

[18] Z. Luo and J. J. Nieto, "New results for the periodic boundary value problem for impulsive integrodifferential equations," Nonlinear Analysis: Theory, Methods $\mathcal{E}$ Applications, vol. 70, no. 6, pp. 2248-2260, 2009.

[19] J. J. Nieto and D. O'Regan, "Variational approach to impulsive differential equations," Nonlinear Analysis: Real World Applications, vol. 10, no. 2, pp. 680-690, 2009.

[20] M. Benchohra, J. Henderson, and S. Ntouyas, Impulsive Differential Equations and Inclusions, vol. 2 of Contemporary Mathematics and Its Applications, Hindawi Publishing Corporation, New York, NY, USA, 2006.

[21] G. Iovane, "Some new integral inequalities of Bellman-Bihari type with delay for discontinuous functions," Nonlinear Analysis: Theory, Methods E Applications, vol. 66, no. 2, pp. 498-508, 2007.

[22] A. Samoilenko, S. Borysenko, C. Cattani, G. Matarazzo, and V. Yasinsky, Differential Models: Stability, Inequalities and Estimates, Naukova Dumka, Kiev, Russia, 2001.

[23] D. S. Borysenko, A. Gallo, and R. Toscano, "Integral inequalities Gronwall-Bellman type for discontinuous functions and estimates of solutions impulsive systems," in Proc.DE@CAS, pp. 5-9, Brest, 2005. 